"This is the pre-peer reviewed version of the following article: AlSharawi, Z., \& Amleh, A. (2017). The impact of constant effort harvesting on the dynamics of a discrete-time contest competition model. Mathematical Methods in the Applied Sciences, 40(18), 6747-6759, which has been published in final form at https://doi.org/10.1002/mma.4487. This article may be used for non-commercial purposes in accordance with Wiley Terms and Conditions for Use of Self-Archived Versions."

\title{
The impact of constant effort harvesting on the dynamics of a discrete-time contest competition model
}

\author{
Ziyad AlSharawi* ${ }^{*}$ and A.M. Amleh $\ddagger$ \\ $\dagger$ Department of Mathematics and Statistics \\ American University of Sharjah, P. O. Box 26666 \\ University City, Sharjah, UAE \\ $\ddagger$ Sciences and Engineering \\ Paris-Sorbonne University Abu Dhabi \\ P. O. Box 38044, Abu Dhabi, U.A.E.
}

August 14, 2017

\begin{abstract}
In this paper, we study a general discrete-time model representing the dynamics of a contest competition species with constant effort exploitation. In particular, we consider the difference equation $x_{n+1}=x_{n} f\left(x_{n-k}\right)-h x_{n}$ where $h>0, k \in\{0,1\}$, and the density dependent function $f$ satisfies certain conditions that are typical of a contest competition. The harvesting parameter $h$ is considered as the main parameter and its effect on the general dynamics of the model is investigated. In the absence of delay in the recruitment $(k=0)$, we show the effect of $h$ on the stability, the maximum sustainable yield, the persistence of solutions and how the intraspecific competition change from contest to scramble competition. When the delay in recruitment is one $(k=1)$, we show that a Neimark-Sacker bifurcation occurs, and the obtained invariant curve is supercritical. Furthermore, we give a characterization of the persistent set.
\end{abstract}

AMS Subject Classification: 39A10, 92D25

Keywords: Contest competition; Scramble competition; Neimark-Sacker bifurcation; Persistence.

\section{Introduction}

Different types of intraspecific competitions are well-known in population ecology among which are contest and scramble competitions $[11,20,27,28,30]$. In contest competition, successful competitors get all they need for survival or reproduction, while non-successful competitors are doomed to their fate. On the other hand, in scramble competition, success is incomplete; resources are shared equally among all competitors. As argued by Nicholson [30], populations of animals which scramble for their governing requisites are difficult to represent mathematically, and therefore, mathematical theories of population regulations are needed. Moreover, the

${ }^{*}$ Corresponding author: zsharawi@aus.edu 
borderline between the two forms of competition may not be sharply defined [4]. For ecological examples of species on both types of competitions, we refer the reader to [11,30,37,38]. In single-species populations, models with compensatory recruitment are commonly the suitable candidates to represent contest competition and the Beverton-Holt model $x_{n+1}=\frac{\mu x_{n}}{r+x_{n}}$ is a typical discrete-time example $[5,11]$. On the other hand, models with overcompensatory recruitment are commonly the suitable candidates to present scramble competition, and the Logistic and Ricker models $\left(x_{n+1}=\mu x_{n}\left(r-x_{n}\right)\right.$ and $x_{n+1}=\mu x_{n} \exp \left(-r x_{n}\right)$, respectively) are considered to be the typical discrete-time examples [11,29,33].

In this paper, we consider a general mathematical form that can quantify the contest competition of a single-species population, then add a constant effort harvesting strategy to the model. In addition, we allow a short time lag in the recruitment. In particular, we consider the (unexploited) difference equation $x_{n+1}=x_{n} f\left(x_{n-k}\right), n=0,1, \ldots$, where the time lag in recruitment is $k \in\{0,1\}$. The form $x f(x)$ is used to stress the zero steady state, and the percapita growth rate $f$ has to be considered based on observations or facts concerning the modeled species. In this paper, the per-capita growth rate function $f$ is assumed to satisfy the following three conditions:

(i) $f$ is sufficiently smooth on $[0, \infty)$.

(ii) $f(0)=b>1$ and $f(t)$ is decreasing.

(iii) $t f(t)$ is increasing and bounded.

Our framed model with the above mathematical assumptions on $f$ encompasses compensatory models such as Beverton-Holt and Pielou's models while avoids overcompensatory models such as the Logistic and Ricker models.

When a population represented by our model is exploited (post reproduction exploitation) at a proportional rate, it is natural to consider a harvesting term of the form $H\left(x_{n}, x_{n-1}\right)=$ $h_{1} x_{n}+h_{2} x_{n-1}, h_{1}, h_{2} \geq 0$. In this paper, we focus on the impact of constant effort harvesting when $h_{2}=0$ and $h_{1}=: h>0$; other variations of pre and post reproduction exploitation may be considered in future work on the subject. For more information on the biological meaning and impact of pre and post reproduction exploitation, we refer the reader to [10,24,26,35,42]. Therefore, we are concerned with the dynamics of the difference equation

$$
x_{n+1}=x_{n} f\left(x_{n-k}\right)-h x_{n}, \quad k=0 \text { or } 1, \quad n \in \mathbb{N}:=\mathbb{Z}^{+} \cup\{0\} \text { and } h>0 .
$$

There has been a growing interest in the impact of various harvesting strategies on populations governed by discrete models. For instance, the impact of constant rate harvesting/stocking on co-existence between competing species in a competitive exclusion system $[3,39]$, the negative impact of harvesting when used to control nuisance and invasive species [42], the impact of constant effort harvesting on controlling chaos and on the hydra effect [24], bubbling and sudden collapse [25] and the optimal impact of various harvesting strategies on species abundance [1,2]. Other notions and results concerning various harvesting strategies can be found in $[7,8,12,14,16]$.

In this theoretical study, we are interested in characterizing the impact of constant effort harvesting on the general dynamics of discrete-time contest competition models of single species with or without time lag in recruitment. The paper is organized as follows: In section two, we present the local stability of equilibrium solutions for both $k=0$ and $k=1$. In section three, we 
focus on Eq.(1.1) with $k=0$ and present results on persistence of solutions and global stability of the positive equilibrium. As the parameter $h$ is varied, we show that the model undergoes a period-doubling bifurcation and chaos. We also present conditions under which the model changes from contest competition to scramble competition. Moreover, conditions on the parameter are given to maximize the average harvest. As an illustrative example, we apply our results to the Beverton-Holt model with constant effort harvesting. In section four, we focus on Eq.(1.1) with $k=1$ and present results on the persistence and oscillation of solutions. Furthermore, we show that as we vary the parameter $h$, a Neimark-Sacker bifurcation occurs and a supercritical invariant curve bifurcates from the positive equilibrium. As an illustrative example, we apply our results to Pielou's difference equation [31,32] with constant effort harvesting. Finally, we close this paper by a short conclusion.

\section{Equilibria and local stability}

Zero is always an equilibrium solution of Eq.(1.1) and when $h<b-1$, a positive equilibrium appears, namely, $\bar{x}_{h}=f^{-1}(1+h)$. When $h>b-1$, Eq.(1.1) gives $x_{n+1}<(b-h) x_{n}$, and therefore, populations are doomed to extinction. Although populations are also depleted at $h=b-1$, we still shed some light on this case as a boundary case; however, throughout this paper, $h<b-1$ is considered as our primary objective. Now, to study the stability of the equilibrium solutions, we linearize Eq.(1.1) for both $k=0$ and $k=1$, i.e.,

$$
\begin{gathered}
x_{n+1}=x_{n}\left(f\left(x_{n}\right)-h\right)=g\left(x_{n}\right), \quad n \in \mathbb{N}, \\
x_{n+1}=x_{n}\left(f\left(x_{n-1}\right)-h\right), \quad n \in \mathbb{N} .
\end{gathered}
$$

In Eq.(2.1), since $g^{\prime}(t)=t f^{\prime}(t)+f(t)-h$, we obtain $g^{\prime}(0)=b-h$ and $g^{\prime}\left(\bar{x}_{h}\right)=\bar{x}_{h} f^{\prime}\left(\bar{x}_{h}\right)+1$. On the other hand, when we linearize Eq.(2.2), the characteristic equation about an equilibrium $\bar{x}$ is given by

$$
\lambda^{2}+(h-f(\bar{x})) \lambda-\bar{x} f^{\prime}(\bar{x})=0 .
$$

Observe that at $\bar{x}=0$, we obtain $\lambda(\lambda-b+h)=0$, while at $\bar{x}=\bar{x}_{h}$, we obtain $\lambda^{2}-\lambda-\bar{x}_{h} f^{\prime}\left(\bar{x}_{h}\right)=0$. Also, keep in mind that $f^{\prime}\left(\bar{x}_{h}\right)<0$. In the following theorem, we use these facts to characterize the local stability of all equilibrium solutions.

Theorem 2.1. Assume $0<h<b-1$. Each of the following holds true:

[A] In Eq.(2.1),

(i) $\bar{x}=0$ is a repeller.

(ii) $\bar{x}_{h}$ is locally asymptotically stable $(L A S)$ when $\bar{x}_{h} f^{\prime}\left(\bar{x}_{h}\right)>-2$, a repeller when $\bar{x}_{h} f^{\prime}\left(\bar{x}_{h}\right)<$ -2 , and is a non-hyperbolic equilibrium when $\bar{x}_{h} f^{\prime}\left(\bar{x}_{h}\right)=-2$.

[B] In Eq.(2.2),

(i) $\bar{x}=0$ is a saddle whose stable manifold is formed along the non-negative $x$-axis.

(ii) $\bar{x}_{h}$ is locally asymptotically stable when $\bar{x}_{h} f^{\prime}\left(\bar{x}_{h}\right)>-1$, unstable when $\bar{x}_{h} f^{\prime}\left(\bar{x}_{h}\right)<-1$, and a non-hyperbolic equilibrium when $\bar{x}_{h} f^{\prime}\left(\bar{x}_{h}\right)=-1$. 
Observe that the condition $\bar{x}_{h} f^{\prime}\left(\bar{x}_{h}\right)=-1$ in Part [B] of Theorem 2.1 is in fact the same as $\max _{t} t(f(t)-h)=\bar{x}_{h}$ over the interval $\left[0, f^{-1}(h)\right]$. In Figure 1, we illustrate Theorem 2.1 for the specific examples

$$
\text { (a) } \quad x_{n+1}=\frac{b x_{n}}{1+x_{n}}-h x_{n} \quad \text { and } \quad \text { (b) } \quad x_{n+1}=\frac{b x_{n}}{1+x_{n-1}}-h x_{n} .
$$

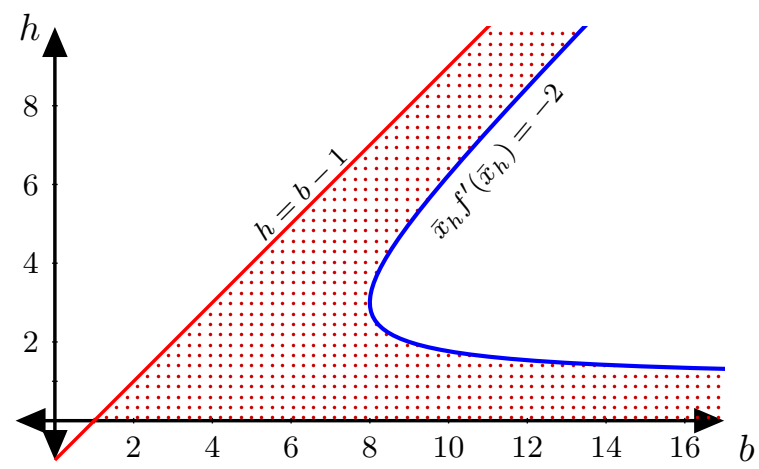

(a) with no delay (i.e., $k=0$ )

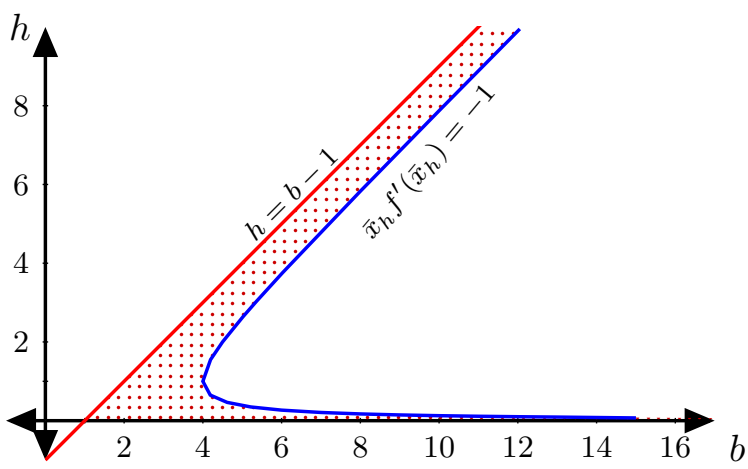

(b) with delay $k=1$

Figure 1: The shaded region in each figure shows the values of $b$ and $h$ that make $\bar{x}_{h}$ positive and locally stable.

\section{The equation with no delay in recruitment}

In this section, we focus on the dynamics of the first-order difference equation

$$
x_{n+1}=x_{n}\left(f\left(x_{n}\right)-h\right)=g\left(x_{n}\right), \quad n \in \mathbb{N} \quad \text { and } \quad 0<x_{0}<f^{-1}(h) .
$$

Our unexploited model(at $h=0$ ) is compensatory as long as $b>1$. Recall that a model is called compensatory in an interval $\mathcal{J}$ that contains a unique positive equilibrium whenever all positive population sizes in the interior of $\mathcal{J}$ approach the positive equilibrium monotonically under the iteration of the map. On the other hand, a model is called overcompensatory in $\mathcal{J}$ if some positive population sizes in the interior of $\mathcal{J}$ overshoot the positive equilibrium under the iteration of the map $[40,41]$. At $h=0$, the positive equilibrium $\bar{x}_{h}$ is globally stable with $(0, \infty)$ as the basin of attraction. Now, for $0<h<b-1$ and for the survival of a population, we must have $0<x_{n}<f^{-1}(h)$ for all $n$. We give simple sufficient conditions for the global stability of the positive equilibrium with respect to the domain $\left(0, f^{-1}(h)\right)$ and discuss the effect of $h$ in switching contest competition to scramble competition. Following this, we give conditions under which the average harvest is maximized and discuss persistence of solutions. We close this section by considering the Beverton-Holt model as an illustrative example.

\subsection{Global stability and the transition to scramble competition}

After knowing the threshold level of harvesting under which the local stability of the positive equilibrium $\bar{x}_{h}=f^{-1}(h+1)$ of Eq.(3.1) is preserved, we are interested in finding sufficient and simple conditions under which $\bar{x}_{h}$ is globally asymptotically stable(GAS) with respect to the 
interval $I=\left(0, f^{-1}(h)\right)$. Moreover, we are interested in the transition that takes place when $h$ is increased.

Proposition 3.1. When $h \leq 1$, the positive equilibrium $\bar{x}_{h}$ of Eq.(3.1) is globally asymptotically stable with respect to the interval $I=\left(0, f^{-1}(h)\right)$.

Proof. Assume $h \leq 1$. It is sufficient to show that Eq.(3.1) has no 2-cycle (see [13] or [15]). By contradiction, suppose that Eq.(3.1) has a 2-cycle, say $\{\phi, \psi\}$ where $\phi \neq \psi$ and $\phi, \psi \in I$. We have

$$
\psi=\phi(f(\phi)-h) \quad \text { and } \quad \phi=\psi(f(\psi)-h) .
$$

Subtract the two equations to obtain

$$
\psi-\phi=\phi f(\phi)-\psi f(\psi)+h(\psi-\phi)
$$

or equivalently,

$$
h-1=\frac{\phi f(\phi)-\psi f(\psi)}{\phi-\psi} .
$$

Now, consider $F(t)=t f(t)$, by the Mean Value theorem, $F^{\prime}(c)=h-1$ for some $c$ between $\phi$ and $\psi$. However, this is not possible since $F(t)$ is increasing.

The first period-doubling bifurcation occurs when $h$ satisfies

$$
\bar{x}_{h} f^{\prime}\left(\bar{x}_{h}\right)=-2
$$

This suggests that $\bar{x}_{h} f^{\prime}\left(\bar{x}_{h}\right)>-2$ is the suitable condition to use for a compensatory dynamics over the interval $I=\left(0, f^{-1}(h)\right)$; however, Proposition 3.1 is tempting for the simplicity of the condition. Although the interval $I$ shrinks as we increase $h$, there is no collapse of populations within $I$ as long as the maximum possible value of $x_{n+1}$ is less than the maximum possible value of $x_{n}$, i.e.,

$$
M_{h}:=\max _{0<x<f^{-1}(h)} g(x)<f^{-1}(h) .
$$

When $h$ satisfies $M_{h}=f^{-1}(h)$, the map $g(x)$ is turbulent. For the definition of turbulent maps and the implications, we refer the reader to [6]. In particular, when $g$ becomes turbulent, cycles of all periods exist. Furthermore, essential extinction occurs at $M_{h}=f^{-1}(h)$, i.e., almost every initial population density leads to extinction [34]. Also, as $h$ increases from 0 , the intraspecific competition changes from contest to scramble competition. Figure 2 illustrates the change in dynamics as we increase $h$.

\subsection{Maximizing the average harvest}

Let $\left\{x_{n}\right\}$ be a positive solution of Eq.(3.1), and define

$$
A v\left(x_{n}\right):=\frac{1}{n+1} \sum_{j=0}^{n} x_{j} .
$$

We are interested in maximizing $h A v\left(x_{n}\right)$ without driving populations within $\left(0, f^{-1}(h)\right)$ to extinction. 
Lemma 3.1. Suppose $F(t)=t f(t)$ is concave. Then

$$
\limsup A v\left(x_{n}\right) \leq \bar{x}_{h}
$$

Proof. Sum both sides of Eq.(3.1) to obtain

$$
(h+1) \sum_{j=0}^{n} x_{j}+x_{n+1}-x_{0}=\sum_{j=0}^{n} F\left(x_{j}\right) .
$$

Divide both sides by $n+1$ and use the fact that $F(t)$ is concave, then apply Jensen's inequality to obtain

$$
(h+1) A v\left(x_{n}\right)+\frac{x_{n+1}-x_{0}}{n+1}=\frac{1}{n+1} \sum_{j=0}^{n} F\left(x_{j}\right) \leq F\left(A v\left(x_{n}\right)\right) .
$$

Take limsup of both sides and use the fact that $F(t)$ is increasing to obtain

$$
(h+1) \lim \sup A v\left(x_{n}\right) \leq F\left(\lim \sup A v\left(x_{n}\right)\right)
$$

Thus,

$$
\limsup A v\left(x_{n}\right) \leq \limsup A v\left(x_{n}\right)\left(f\left(\lim \sup A v\left(x_{n}\right)\right)-h\right),
$$

and consequently $\lim \sup A v\left(x_{n}\right) \leq \bar{x}_{h}$ as required.

The following theorem gives sufficient conditions for maximizing the average harvest.

Theorem 3.1. Suppose $F(t)=t f(t)$ is concave. The average harvest in Eq.(3.1) can be maximized when $h$ satisfies either $\bar{x}_{h} f^{\prime}\left(\bar{x}_{h}\right)=-h$ or $\bar{x}_{h} f^{\prime}\left(\bar{x}_{h}\right)=-2$.

Proof. From Lemma 3.1, we maximize the harvest by preserving the global stability of the positive equilibrium and maximizing $h \bar{x}_{h}$, or at the bifurcation point when a 2-cycle is born. In the first case, we maximize $h \bar{x}_{h}=h f^{-1}(h+1)$, and the extreme value takes place at $\bar{x}_{h} f^{\prime}\left(\bar{x}_{h}\right)=$ $-h$. At the second case, the equilibrium bifurcates into a 2 -cycle when $\bar{x}_{h} f^{\prime}\left(\bar{x}_{h}\right)=-2$.

\subsection{Persistence}

A solution $\left\{x_{n}\right\}_{n=-1}^{\infty}$ of Eq.(3.1) (or Eq.(4.1)) is called persistent if $x_{n}>0$ for all $n$. It is called strongly persistent if it is persistent and $\liminf x_{n}>0$. It is worth mentioning here that several concepts such as permanence and uniform persistence are used in the literature [21,34]. However, throughout this paper, we use persistent/strongly persistent. The set of all initial conditions that give persistent solutions is called the persistent set. Similarly, we can define the strongly persistent set. We use $\mathcal{D}_{h}$ for the persistent set and $\mathcal{D}_{h}^{*}$ for the strongly persistent set at harvesting level $h$. As observed in Section 2, when $h \geq b-1$, the population is doomed to extinction. However, according to our definition, we have the possibility of persistence but not strong persistence. Indeed, if $h>b-1$ in Eq.(3.1), then $\mathcal{D}_{h}=\mathcal{D}_{h}^{*}=\emptyset$. If $h=b-1$, then $\mathcal{D}_{h}=\left(0, \frac{1}{b-1}\right)$ while $\mathcal{D}_{h}^{*}=\emptyset$. If $h<b-1$ and $\max _{t} t(f(t)-h)<f^{-1}(h)$ for $0 \leq t \leq f^{-1}(h)$, then $\mathcal{D}_{h}=\mathcal{D}_{h}^{*}=\left(0, f^{-1}(h)\right)$. 


\subsection{The Beverton-Holt model with constant effort harvesting}

As an illustrative example of Eq.(3.1), we consider the Beverton-Holt model which is given by the first-order difference equation

$$
y_{n+1}=\frac{b K y_{n}}{K+(b-1) y_{n}}, \quad n \in \mathbb{N},
$$

where the parameter $b>1$ represents the population growth rate and $K$ is the carrying capacity of the environment. Forcing constant effort harvesting on Eq.(3.3) gives

$$
y_{n+1}=\frac{b K y_{n}}{K+(b-1) y_{n}}-h y_{n}
$$

To reduce the number of parameters, let $x_{n}=\frac{b-1}{K} y_{n}$, we obtain

$$
x_{n+1}=\frac{b x_{n}}{1+x_{n}}-h x_{n}, \quad n \in \mathbb{N} .
$$

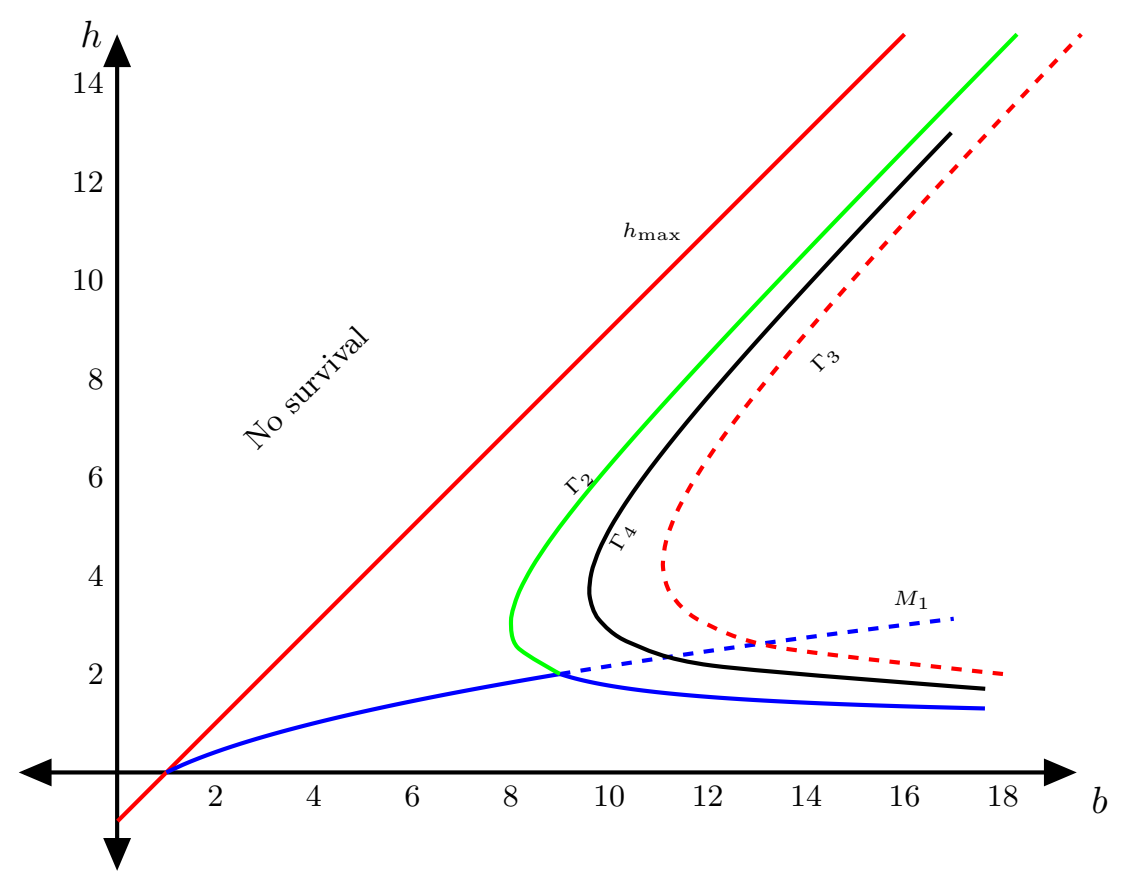

Figure 2: This figure clarifies the effect of the parameters $b$ and $h$ on the population survival, bifurcation and maximum total harvesting. The curves are given by $h_{\max }: h=b-1, M_{1}$ : $h=\sqrt{b}-1, \Gamma_{2}: b(h-1)=(h+1)^{2}, \Gamma_{3}: b(h-1)^{2}=h(h+1)^{2}$ and $\Gamma_{4}: b(h-1)^{2}=$ $h^{3}-(h-1)^{2}+\sqrt{h(3 h-2)\left(2 h^{2}-2 h+1\right)}$.

Based on the first part of Theorem 2.1, we check the condition $\bar{x}_{h} f^{\prime}\left(\bar{x}_{h}\right)=-2$ at the positive equilibrium $\bar{x}_{h}=\frac{b}{h+1}-1$. We find the condition $h^{2}+(2-b) h+b+1>0$ that makes $\bar{x}_{h}$ LAS, while the reversed inequality makes $\bar{x}_{h}$ a repeller. Next, we use Proposition 3.1 to obtain $\bar{x}_{h}$ globally asymptotically stable with respect to the interval $I=\left(0, \frac{b}{h}-1\right)$ whenever $h \leq \min \{1, b-1\}$. However, since the conditions of Proposition 3.1 are sufficient conditions, we can obtain a stronger result. Indeed, if we assure that $\left(0, f^{-1}(h)\right)$ is invariant, $\bar{x}_{h}>0$ and no 
periodic solutions of period two exist, then $\bar{x}_{h}$ is globally asymptotically stable with respect to $\left(0, f^{-1}(h)\right)$. Thus, after some computations, we obtain the necessary and sufficient conditions on the parameters that make $\bar{x}_{h}$ GAS with respect to $\left(0, f^{-1}(h)\right)$. It is the region in the positive quadrant between the curves of $h_{\max }$ and $\Gamma_{2}$ in Figure 2. It is worth emphasizing that the first period-doubling bifurcation(the birth of a 2-cycle) occurs when Eq.(3.2) is satisfied, that is, when $h^{2}+(2-b) h+b+1=0$. Also, it is a computational matter to find that a second period-doubling bifurcation(the birth of a 4-cycle) occurs at

$$
(h-1)^{2} b^{2}+2\left((h-1)^{2}-h^{3}\right) b+\left(h^{2}-1\right)^{2}=0 .
$$

Finally, the map defined by Eq.(3.4) becomes turbulent when $\max g(x)=\frac{b}{h}-1$. This is given by the $\Gamma_{3}$ curve in Figure 2 .

\section{The equation with a delay in recruitment}

In this section, we focus on the dynamics of the second-order difference equation

$$
x_{n+1}=x_{n}\left(f\left(x_{n-1}\right)-h\right), \quad n \in \mathbb{N} \quad \text { and } \quad 0 \leq x_{-1}, x_{0} \leq f^{-1}(h) .
$$

In particular, we show the existence of a Neimark-Sacker bifurcation in which the obtained curve is supercritical, then we discuss persistence of solutions. As an illustrative example, we discuss Pielou's model $[31,32]$ with constant effort harvesting.

\subsection{Neimark-Sacker bifurcation}

In Theorem 2.1, we found that when $h$ satisfies $\bar{x}_{h} f^{\prime}\left(\bar{x}_{h}\right)=-1$, the positive equilibrium of Eq.(4.1) is non-hyperbolic. Here, we find conditions under which a Neimark-Sacker bifurcation occurs together with a supercritical invariant curve. To establish this, we start by putting Eq.(4.1) into system form. Set $x_{n-1}=y_{n}$ to obtain

$$
\left\{\begin{array}{l}
x_{n+1}=x_{n}\left(f\left(y_{n}\right)-h\right) \\
y_{n+1}=x_{n}
\end{array}\right.
$$

Lemma 4.1. Let $m_{h}=-\bar{x}_{h} f^{\prime}\left(\bar{x}_{h}\right)$. Assume that $\max _{h} m_{h}>1 / 4$, then there is an interval $\left[h_{1}, h_{2}\right]$ such that the eigenvalues of the linearized system are non-real for all $h \in\left[h_{1}, h_{2}\right]$. Furthermore, there exists $\tilde{h} \in\left[h_{1}, h_{2}\right]$ such that the non-real eigenvalues cross the unit circle at $h=\tilde{h}$.

Proof. From the assumptions $(i),(i i)$ and $(i i i)$ on $f(t), \bar{x}_{h}=f^{-1}(1+h)$ is decreasing in $h$ and $\bar{x}_{h} \in\left[0, f^{-1}(h)\right]$. Also, $m_{h}=-\bar{x}_{h} f^{\prime}\left(\bar{x}_{h}\right)$ is continuous in $h$ and

$$
m_{0}=-\bar{x}_{0} f^{\prime}\left(\bar{x}_{0}\right)=-f^{-1}(1) f^{\prime}\left(f^{-1}(1)\right)
$$

while

$$
m_{b-1}=-\bar{x}_{b-1} f^{\prime}\left(\bar{x}_{b-1}\right)=-f^{-1}(b) f^{\prime}\left(f^{-1}(b)\right)=0
$$


The eigenvalues are complex numbers when $m_{h}>\frac{1}{4}$ and they cross the unit circle when $m_{h}=1$. Now, the continuity of $m_{h}$ and the given assumption give us the desired conclusion.

Next, we focus on the bifurcation that occurs at $h=\tilde{h}$.

Theorem 4.1. Consider Eq.(4.1) with $f \in C^{2}([0, \infty))$ and $h<b-1$. Assume $\max _{h} m_{h}>\frac{1}{4}$. If $\bar{x}_{\tilde{h}}^{2} f^{\prime \prime}\left(\bar{x}_{\tilde{h}}\right) \neq 1$, then a Neimark-Sacker bifurcation occurs at $h=\tilde{h}$.

Proof. Consider $\left[h_{1}, h_{2}\right]$ to be the interval assured by Lemma 4.1. The eigenvalues of the linearized equation about $\bar{x}_{h}$ are

$$
\lambda_{1,2}=\frac{1}{2}\left(1 \pm \sqrt{4 m_{h}-1} i\right)=\sqrt{m_{h}} e^{ \pm i \theta_{h}}
$$

where $\tan \left(\theta_{h}\right)=\sqrt{4 m_{h}-1}$. At $h=\tilde{h}$, we have $m_{\tilde{h}}=1$, and therefore, the eigenvalues cross the unit circle at $\theta_{\tilde{h}}=\frac{\pi}{3}$. Hence,

$$
e^{i k \theta_{\tilde{h}}} \neq 1 \quad \text { for } \quad k=1,2,3,4 .
$$

Next, we use the fact $\left|\lambda_{1,2}\right|^{2}=m_{h}$, to show that $\frac{d}{d h} m_{\tilde{h}} \neq 0$. We have

$$
\begin{aligned}
\frac{d}{d h} m_{h} & =-\bar{x}_{h}^{\prime}\left(f^{\prime}\left(\bar{x}_{h}\right)+\bar{x}_{h} f^{\prime \prime}\left(\bar{x}_{h}\right)\right) \\
& =-\frac{\bar{x}_{h}^{\prime}}{\bar{x}_{h}}\left(\bar{x}_{h} f^{\prime}\left(\bar{x}_{h}\right)+\bar{x}_{h}^{2} f^{\prime \prime}\left(\bar{x}_{h}\right)\right) \\
\frac{d}{d h} m_{\tilde{h}} & =-\frac{\bar{x}_{\tilde{h}}^{\prime}}{\bar{x}_{\tilde{h}}}\left(\bar{x}_{\tilde{h}} f^{\prime}\left(\bar{x}_{\tilde{h}}\right)+\bar{x}_{\tilde{h}}^{2} f^{\prime \prime}\left(\bar{x}_{\tilde{h}}\right)\right) \\
& =-\frac{\bar{x}_{\tilde{h}}^{\prime}}{\bar{x}_{\tilde{h}}}\left(-1+\bar{x}_{\tilde{h}}^{2} f^{\prime \prime}\left(\bar{x}_{\tilde{h}}\right)\right) .
\end{aligned}
$$

Because $\bar{x}_{h}$ is strictly decreasing in $h$, then $\bar{x}_{h}^{\prime} \neq 0$. Thus, $\bar{x}_{\tilde{h}}^{2} f^{\prime \prime}\left(\bar{x}_{\tilde{h}}\right) \neq 1$ guarantees that $\frac{d}{d h} m_{\tilde{h}} \neq 0$ as desired.

After establishing the conditions in which a Neimark-Sacker Bifurcation occurs, we proceed to investigate the nature of this bifurcation (supercritical or subcritical $[18,19,23]$ ). We shift the equilibrium $\left(\bar{x}_{h}, \bar{x}_{h}\right)$ of System (4.2) to the origin by considering the change of variables $u_{n}=x_{n}-\bar{x}_{h}$ and $v_{n}=y_{n}-\bar{x}_{h}$. We obtain the new system

$$
\left\{\begin{array}{l}
u_{n+1}=\left(u_{n}+\bar{x}_{h}\right)\left(f\left(v_{n}+\bar{x}_{h}\right)-h\right)-\bar{x}_{h} \\
v_{n+1}=u_{n} .
\end{array}\right.
$$

Now, assume $f$ is sufficiently smooth, and use Taylor expansion about $(0,0)$ to obtain

$$
\left[\begin{array}{l}
u_{n+1} \\
v_{n+1}
\end{array}\right]=J(0,0)\left[\begin{array}{l}
u_{n} \\
v_{n}
\end{array}\right]+\left[\begin{array}{c}
G_{1}\left(u_{n}, v_{n}\right) \\
0
\end{array}\right],
$$

where

$$
G_{1}\left(u_{n}, v_{n}\right)=f^{\prime}\left(\bar{x}_{h}\right) u_{n} v_{n}+\left(u_{n}+\bar{x}_{h}\right) \sum_{k=2}^{\infty} f^{(k)}\left(\bar{x}_{h}\right) \frac{v_{n}^{k}}{k !} .
$$


For our writing convenience, define $q:=\sqrt{4 m_{h}-1}$. We transform the system and put the linear part in Jordan normal form by considering

$$
\left[\begin{array}{l}
u_{n} \\
v_{n}
\end{array}\right]=Q\left[\begin{array}{l}
U_{n} \\
V_{n}
\end{array}\right], \quad \text { where } \quad Q:=\frac{1}{2}\left[\begin{array}{cc}
1 & -q \\
2 & 0
\end{array}\right] .
$$

In this case, we obtain

$$
\begin{aligned}
{\left[\begin{array}{c}
U_{n+1} \\
V_{n+1}
\end{array}\right] } & =\frac{1}{2}\left[\begin{array}{cc}
1 & -q \\
q & 1
\end{array}\right]\left[\begin{array}{c}
U_{n} \\
V_{n}
\end{array}\right]+Q^{-1}\left[\begin{array}{c}
G_{1}\left(\frac{1}{2} U_{n}-\frac{1}{2} q V_{n}, U_{n}\right) \\
0
\end{array}\right] \\
& =\frac{1}{2}\left[\begin{array}{cc}
1 & -q \\
q & 1
\end{array}\right]\left[\begin{array}{c}
U_{n} \\
V_{n}
\end{array}\right]+\left[\begin{array}{c}
F_{1}\left(U_{n}, V_{n}\right) \\
F_{2}\left(U_{n}, V_{n}\right)
\end{array}\right]
\end{aligned}
$$

where $F_{1}\left(U_{n}, V_{n}\right)=0$ and

$$
\begin{aligned}
F_{2}\left(U_{n}, V_{n}\right) & =\frac{-2}{q} G_{1}\left(\frac{1}{2} U_{n}-\frac{1}{2} q V_{n}, U_{n}\right) \\
& =V_{n} \sum_{k=1}^{\infty} f^{(k)}\left(\bar{x}_{h}\right) \frac{U_{n}^{k}}{k !}-\frac{1}{q} \sum_{k=2}^{\infty}\left(k f^{(k-1)}\left(\bar{x}_{h}\right)+2 \bar{x}_{h} f^{(k)}\left(\bar{x}_{h}\right)\right) \frac{U_{n}^{k}}{k !} .
\end{aligned}
$$

Thus, we need to investigate the expression

$$
A(h)=\operatorname{Re}\left(\frac{\left(1-2 e^{i \theta_{h}}\right) e^{-2 i \theta_{h}}}{1-e^{i \theta_{h}}} C_{11} C_{20}\right)+\frac{1}{2}\left|C_{11}\right|^{2}+\left|C_{02}\right|^{2}-R e\left(e^{-i \theta_{h}} C_{21}\right),
$$

where

$$
\begin{aligned}
C_{20} & =\frac{1}{8}\left[F_{1 U U}-F_{1 V V}+2 F_{2 U V}+i\left(F_{2 U U}-F_{2 V V}-2 F_{1 U V}\right)\right] \\
& =\frac{1}{4} f^{\prime}\left(\bar{x}_{h}\right)-\frac{1}{4 q}\left(f^{\prime}\left(\bar{x}_{h}\right)+\bar{x}_{h} f^{\prime \prime}\left(\bar{x}_{h}\right)\right) i, \\
C_{11} & =\frac{1}{4}\left[F_{1 U U}+F_{1 V V}+i\left(F_{2 U U}+F_{2 V V}\right)\right] \\
& =-\frac{1}{2 q}\left(f^{\prime}\left(\bar{x}_{h}\right)+\bar{x}_{h} f^{\prime \prime}\left(\bar{x}_{h}\right)\right) i, \\
C_{02} & =\frac{1}{8}\left[F_{1 U U}-F_{1 V V}-2 F_{2 U V}+i\left(F_{2 U U}-F_{2 V V}+2 F_{1 U V}\right)\right] \\
& =-\frac{1}{4} f^{\prime}\left(\bar{x}_{h}\right)-\frac{1}{4 q}\left(f^{\prime}\left(\bar{x}_{h}\right)+\bar{x}_{h} f^{\prime \prime}\left(\bar{x}_{h}\right)\right) i,
\end{aligned}
$$




$$
\begin{aligned}
C_{21} & =\frac{1}{16}\left[F_{1 U U U}-F_{1 U V V}+F_{2 U U V}+F_{2 V V V}+i\left(F_{2 U U U}+F_{2 U V V}-F_{1 U U V}-F_{1 V V V}\right)\right] \\
& =\frac{1}{16} f^{\prime \prime}\left(\bar{x}_{h}\right)-\frac{1}{16 q}\left(3 f^{\prime \prime}\left(\bar{x}_{h}\right)+2 \bar{x}_{h} f^{\prime \prime \prime}\left(\bar{x}_{h}\right)\right) i .
\end{aligned}
$$

Recall that at $h=\tilde{h}$, we have $\theta_{\tilde{h}}=\frac{\pi}{3}$. Thus,

$$
\frac{\left(1-2 e^{i \theta_{h}}\right) e^{-2 i \theta_{h}}}{1-e^{i \theta_{h}}}=\frac{-1}{2}(3+i \sqrt{3})
$$

and we are ready to give the following result:

Theorem 4.2. Consider Eq.(4.1) with $f \in C^{4}([0, \infty))$ and $h<b-1$. If $\max _{h} m_{h}>1, \bar{x}_{h}^{2} f^{\prime \prime}\left(\bar{x}_{h}\right) \neq$ 1 and $\bar{x}_{h}^{3} f^{\prime \prime \prime}\left(\bar{x}_{h}\right)>-\frac{3}{2}$, then the invariant curve obtained by the Neimark-Sacker Bifurcation at $h=\tilde{h}$ is supercritical.

Proof. It remains to show that the bifurcation is supercritical. At $h=\tilde{h}$, we obtain

$$
\begin{aligned}
\operatorname{Re}\left(\frac{\left(1-2 e^{i \theta_{\tilde{h}}}\right) e^{-2 i \theta_{\tilde{h}}}}{1-e^{i \theta_{\tilde{h}}}} C_{11} C_{20}\right) & =-\frac{1}{2} \operatorname{Re}\left((3+\sqrt{3} i) C_{11} C_{20}\right) \\
& =\frac{1}{16 q^{2}}\left(3 \bar{x}_{\tilde{h}} f^{\prime \prime}\left(\bar{x}_{\tilde{h}}\right)+(3-\sqrt{3} q) f^{\prime}\left(\bar{x}_{\tilde{h}}\right)\right)\left(\bar{x}_{\tilde{h}} f^{\prime \prime}\left(\bar{x}_{\tilde{h}}\right)+f^{\prime}\left(\bar{x}_{\tilde{h}}\right)\right), \\
\frac{1}{2}\left|C_{11}\right|^{2}+\left|C_{02}\right|^{2} & =\frac{3}{16 q^{2}}\left(\bar{x}_{\tilde{h}} f^{\prime \prime}\left(\bar{x}_{\tilde{h}}\right)+f^{\prime}\left(\bar{x}_{\tilde{h}}\right)\right)^{2}+\frac{1}{16}\left(f^{\prime}\left(\bar{x}_{\tilde{h}}\right)\right)^{2}
\end{aligned}
$$

and

$$
\operatorname{Re}\left(e^{-i \theta_{\tilde{h}}} C_{21}\right)=-\frac{1}{16}\left(f^{\prime \prime}\left(\bar{x}_{\tilde{h}}\right)+\bar{x}_{\tilde{h}} f^{\prime \prime \prime}\left(\bar{x}_{\tilde{h}}\right)\right) .
$$

Substitute and recall that $q=\sqrt{3}$ at $h=\tilde{h}$ to obtain

$$
16 A(\tilde{h})=2\left(f^{\prime}\left(\bar{x}_{\tilde{h}}\right)\right)^{2}+\bar{x}_{\tilde{h}} f^{\prime \prime \prime}\left(\bar{x}_{\tilde{h}}\right)+2\left(\bar{x}_{\tilde{h}}\right)^{2}\left(f^{\prime \prime}\left(\bar{x}_{\tilde{h}}\right)\right)^{2}+3 \bar{x}_{\tilde{h}} f^{\prime}\left(\bar{x}_{\tilde{h}}\right) f^{\prime \prime}\left(\bar{x}_{\tilde{h}}\right)+f^{\prime \prime}\left(\bar{x}_{\tilde{h}}\right) .
$$

Now, multiply both sides by $\left(\bar{x}_{\tilde{h}}\right)^{2}$, and use the fact that $\bar{x}_{\tilde{h}} f^{\prime}\left(\bar{x}_{\tilde{h}}\right)=-1$ to obtain

$$
\begin{aligned}
16\left(\bar{x}_{\tilde{h}}\right)^{2} A(\tilde{h}) & =\left(\bar{x}_{\tilde{h}}\right)^{3} f^{\prime \prime \prime}\left(\bar{x}_{\tilde{h}}\right)+2\left(\left(\left(\bar{x}_{\tilde{h}}\right)^{2} f^{\prime \prime}\left(\bar{x}_{\tilde{h}}\right)\right)^{2}-\left(\bar{x}_{\tilde{h}}\right)^{2} f^{\prime \prime}\left(\bar{x}_{\tilde{h}}\right)+1\right) \\
& \geq\left(\bar{x}_{\tilde{h}}\right)^{3} f^{\prime \prime \prime}\left(\bar{x}_{\tilde{h}}\right)+\frac{3}{2} .
\end{aligned}
$$

Since $\bar{x}_{\tilde{h}}^{3} f^{\prime \prime \prime}\left(\bar{x}_{\tilde{h}}\right)>-3 / 2$, we obtain $A(\tilde{h})>0$ and consequently, the obtained invariant curve is supercritical.

\subsection{Persistence}

Our earlier definition of persistence (strong persistence) is still valid here; however, the sets $\mathcal{D}_{h}$ and $\mathcal{D}_{h}^{*}$ are two dimensional subsets of the positive quadrant including its boundary. As before, $h>b-1$ makes $\mathcal{D}_{h}=\mathcal{D}_{h}^{*}=\emptyset$. At $h=b-1$, populations are depleted which gives $\mathcal{D}_{h}^{*}=\emptyset$; but 
$\mathcal{D}_{h} \neq \emptyset$. Define $T(x, y)=(y, y(f(x)-h))$, then the iterates of the map $T$ help us to visualize the orbits in the positive quadrant, and consequently characterize the dynamics of Eq.(4.1).

Proposition 4.1. Let $h=b-1$ in Eq.(4.1). We obtain

$$
\mathcal{D}_{h}=\left\{(x, y): 0<x, y<f^{-1}(h)\right\} \cup\left\{(0, y): 0<y<f^{-1}(h)\right\} \quad \text { and } \quad \mathcal{D}_{h}^{*}=\emptyset \text {. }
$$

Proof. If $(x, y)$ belongs to the positive quadrant and does not belong to the closure of $\mathcal{D}_{h}$, then $T(x, y)$ or $T^{2}(x, y) \notin \mathbb{R}^{+2}$. If $(x, y)$ belongs to the upper, lower or right-hand boundary of $\mathcal{D}_{h}$, then $T^{3}(x, y)=(0,0)$. Finally, take $(x, y) \in \mathcal{D}_{h}$, since $x_{n+1}>0$ whenever $x_{n}>0$ and $0<x<f^{-1}(h)$, we obtain by induction $T^{n}(x, y) \in \mathcal{D}_{h}$ for all $n$. Also, from the fact that $x_{n+1} \leq(b-h) x_{n}=x_{n},\left\{x_{n}\right\}$ is eventually decreasing, and we obtain $\lim _{n \rightarrow \infty} T^{n}(x, y)=(0,0)$, which completes the proof.

Next, define the set $\mathcal{S}_{0}$ to be $\mathcal{D}_{h}$ at $h=b-1$, i.e., $\mathcal{S}_{0}:=\mathcal{D}_{b-1}$. Since initial points out of the square $\mathcal{S}_{0}$ are mapped out of the positive quadrant in finite time, we must have $\mathcal{D}_{h}^{*} \subseteq \mathcal{D}_{h} \subseteq \mathcal{S}_{0}$. Therefore, we limit our attention to the square $\mathcal{S}_{0}$ as the domain of the map $T$. Define the function $y^{*}$ as

$$
y^{*}(x)=\frac{f^{-1}(h)}{f(x)-h}, \quad 0 \leq x<f^{-1}(h) .
$$

Because of our need in the sequel, we summarize some properties of $y^{*}$ in the following lemma.

Lemma 4.2. Let $h \leq b-1$, and assume that $t(f(t)-h)$ is increasing on $\left[0, \bar{x}_{h}\right]$. Each of the following holds true for the function $y^{*}$ defined in Eq.(4.5).

(i) $y^{*}$ is strictly increasing on its domain.

(ii) $y^{*}(0)=\frac{f^{-1}(h)}{b-h}<f^{-1}(h)$ and $y^{*}\left(\bar{x}_{h}\right)=f^{-1}(h)$.

(iii) $T\left(x, y^{*}(x)\right)=\left(y^{*}(x), f^{-1}(h)\right)$.

(iv) $(b-h) x \leq y^{*}(x)$ for all $0 \leq x \leq \bar{x}_{h}$.

Proof. Parts $(i),(i i)$ and $(i i i)$ are obvious. To verify Part $(i v)$, observe that $(b-h) x \leq y^{*}(x)$ is in fact $x(f(x)-h) \leq \frac{f^{-1}(h)}{b-h}$.

Next, we find the persistent set when $0<h<b-1$.

Theorem 4.3. Let $0<h<b-1$, and assume $t(f(t)-h)$ is increasing on the interval $\left[0, \bar{x}_{h}\right]$. The persistent set is given by

$$
\mathcal{D}_{h}=\left\{(x, y): 0 \leq x<f^{-1}(h), 0<y<\min \left\{y^{*}(x), f^{-1}(h)\right\}\right\} .
$$

Proof. Define $\mathcal{R}_{0}:=\left\{(x, y): 0 \leq x<f^{-1}(h), 0<y<\min \left\{y^{*}(x), f^{-1}(h)\right\}\right\}$, and let $\overline{\mathcal{R}_{0}}$ denotes $\mathcal{R}_{0}$ union its boundary. The proof is a little technical, but Fig. 3 makes the idea simple. First, we show that $\mathcal{D}_{h} \subseteq \overline{\mathcal{R}_{0}}$. Since $T(x, y)=(y, y(f(x)-h)), x$ or $y(f(x)-h)$ larger than $f^{-1}(h)$ leads to extinction, and therefore, we must have $\mathcal{D}_{h} \subseteq \overline{\mathcal{R}_{0}}$. Next, we show that $T\left(\overline{\mathcal{R}_{0}}\right)=\overline{\mathcal{R}_{0}}$. Because

$$
T\left(\overline{\mathcal{R}_{0}}\right)=\left\{(y, y(f(x)-h)): 0 \leq x \leq f^{-1}(h), 0 \leq y \leq \min \left\{y^{*}(x), f^{-1}(h)\right\}\right\},
$$


then we need to show that $0<\widetilde{G}(x, y):=y(f(x)-h) \leq \min \left\{f^{-1}(h), y^{*}(x)\right\}$. It is obvious that $\widetilde{G}(x, y)>0$ and $\widetilde{G}$ takes its extreme values on the boundary of $\mathcal{R}_{0}$ (notice that $\operatorname{grad}(\widetilde{G}) \neq 0$ ). Thus, it is enough to check $\widetilde{G}(x, y)$ on the boundary of $\mathcal{R}_{0}$. Define $S_{j}, j=0, \ldots, 4$ as follows.

$$
\begin{aligned}
& S_{0}:=\left\{(0, y): 0<y<\frac{f^{-1}(h)}{b-h}\right\} \\
& S_{1}=\left\{\left(x, y^{*}(x)\right): 0 \leq x \leq \bar{x}_{h}\right\} \\
& S_{2}=\left\{\left(x, f^{-1}(h)\right): \bar{x}_{h} \leq x \leq f^{-1}(h)\right\} \\
& S_{3}=\left\{\left(f^{-1}(h), y\right): 0 \leq y \leq f^{-1}(h)\right\} \\
& S_{4}=\left\{(x, 0): 0 \leq x \leq f^{-1}(h)\right\} .
\end{aligned}
$$

Because $\widetilde{G}\left(S_{4}\right)=0, \widetilde{G}\left(S_{3}\right)=y\left(f\left(f^{-1}(h)\right)-h\right)=0$ and $\widetilde{G}\left(S_{1}\right)=y^{*}(x)(f(x)-h)=f^{-1}(h)$, then $T\left(S_{4}\right)=\{(0,0)\}, T\left(S_{3}\right)=S_{4}$ and $T\left(S_{1}\right) \subseteq S_{2}$, respectively. Next, observe that when $(x, y) \in S_{2}, \widetilde{G}(x, y)=f^{-1}(h)(f(x)-h) \leq f^{-1}(h)$, which implies $T\left(S_{2}\right)=S_{3}$. Finally, when $(x, y) \in S_{0}, \widetilde{G}(x, y)=y(f(0)-h)=y(b-h)<f^{-1}(h)$, which implies together with Lemma 4.2 that $T\left(S_{0}\right)$ belongs to the interior of $\mathcal{R}_{0}$ except the endpoints mapped to the boundary. Up to this end, we proved that $T\left(\overline{\mathcal{R}_{0}}\right)=\overline{\mathcal{R}_{0}}$, and except $S_{0}$, the boundary of $\mathcal{R}_{0}$ is mapped to the saddle equilibrium $(0,0)$ in finite time. Therefore, $\mathcal{D}_{h} \subseteq \mathcal{R}_{0}$. Finally, by tracing back the stable manifold of the origin, we find that $S_{1} \cup S_{2} \cup S_{3} \cup S_{4}$ are the only points in $\subseteq \overline{\mathcal{R}_{0}}$ that are attracted to the origin in finite time. Thus, $\mathcal{D}_{h} \supseteq \mathcal{R}_{0}$, and the proof is complete.

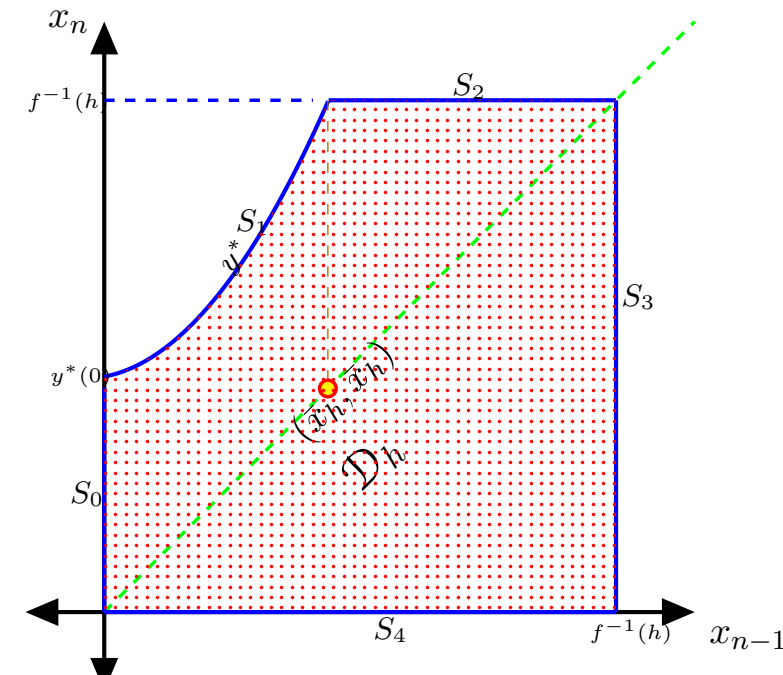

(a) $\mathcal{D}_{h}$ is shaded.

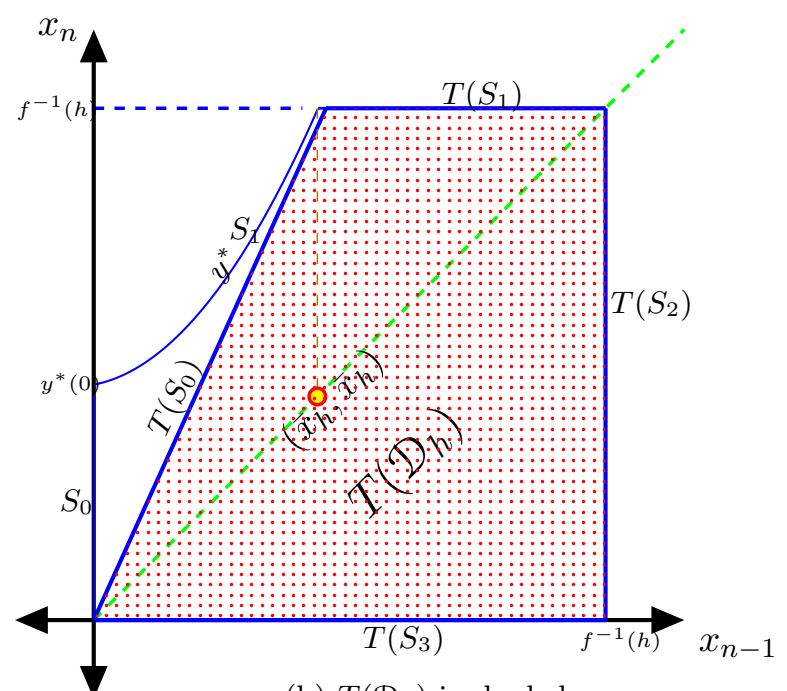

(b) $T\left(\mathcal{D}_{h}\right)$ is shaded

Figure 3: Both figures are given under the assumptions $0<h<b-1$ and $t(f(t)-h)$ is increasing on $\left[0, \bar{x}_{h}\right]$. Also, the invariant regions are given with respect to the map $T\left(x_{n-1}, x_{n}\right)=\left(x_{n}, x_{n+1}\right)$.

Remark 4.1. From the proof of Theorem 4.3, it can be shown that $T\left(\mathcal{D}_{h}\right)$ is an invariant set as well. Also, it becomes obvious that $S_{1} \cup S_{2} \cup S_{3} \cup S_{4}$ forms the basin of attraction of the saddle equilibrium $(0,0)$. On the other hand, finding the basin of attraction of the positive equilibrium is a worthwhile task; however, because of the technicalities and the abstract machinery needed, we leave the problem for a more theoretical paper on the subject. 


\subsection{Pielou's equation with constant effort harvesting}

Pielou's model $[31,32]$ is given by $x_{n+1}=x_{n} f\left(x_{n-k}\right)$, where $f(t)=\frac{b}{1+t}$. To keep things within the context of our developed results, we consider $k=1, b>1$ and force constant effort harvesting, i.e., we consider

$$
x_{n+1}=\frac{b x_{n}}{1+x_{n-1}}-h x_{n}=x_{n}\left(\frac{b}{1+x_{n-1}}-h\right), \quad n \in \mathbb{N} .
$$

As before, we proceed under the assumption that $h \leq b-1$. The local asymptotic stability of the equilibrium solutions is characterized by Theorem 2.1. In particular, the positive equilibrium $\bar{x}_{h}=\frac{b}{h+1}-1$ is LAS when $h^{2}+(2-b) h+1>0$, and a repeller when the inequality is reversed. At $h^{2}+(2-b) h+1=0$, we apply Theorem 4.2 to obtain a Neimark-Sacker bifurcation. Indeed, we clarify this fact in the following result.

Corollary 4.1. Consider $h$ as the varying parameter, and suppose $h<b-1$. Eq.(4.6) undergoes a Neimark-Sacker bifurcation at $h^{2}+(2-b) h+1 \geq 0$, and the obtained invariant curve is supercritical.

Proof. Eq.(4.6) is of the form $x_{n+1}=x_{n}\left(f\left(x_{n-1}\right)-h\right)$. Now,

$$
m_{h}=-\bar{x}_{h} f^{\prime}\left(\bar{x}_{h}\right)=(1+h)\left(1-\frac{h+1}{b}\right)>1 \quad \text { when } \quad b>\frac{(h+1)^{2}}{h} .
$$

Because $h$ starts at zero then increases, we $\tilde{h}$ to be the lower branch of $b h=(1+h)^{2}$, i.e.,

$$
\tilde{h}:=-1+\frac{b}{2}-\frac{1}{2} \sqrt{b(b-4)}, \quad b>4 .
$$

Next, to guarantee $\frac{d}{d h} m_{\tilde{h}} \neq 0$, we investigate the condition $\bar{x}_{\tilde{h}}^{2} f^{\prime \prime}\left(\bar{x}_{\tilde{h}}\right) \neq 1$. Since

$$
f^{\prime \prime}(t)=\frac{2 b}{(1+t)^{3}}=\frac{-2}{b} f(t) f^{\prime}(t)
$$

then

$$
\begin{aligned}
\bar{x}_{h}^{2} f^{\prime \prime}\left(\bar{x}_{h}\right) & =\frac{-2}{b}\left(\bar{x}_{h} f\left(\bar{x}_{h}\right)\right)\left(\bar{x}_{h} f^{\prime}\left(\bar{x}_{h}\right)\right) \\
& \left.=\frac{2}{b}\left(\bar{x}_{h} f\left(\bar{x}_{h}\right)\right) m_{h}\right) \\
& =\frac{2}{b}(1+h) \bar{x}_{h} m_{h} .
\end{aligned}
$$

Therefore,

$$
\bar{x}_{\tilde{h}}^{2} f^{\prime \prime}\left(\bar{x}_{\tilde{h}}\right)=\frac{2}{b}(1+\tilde{h}) \bar{x}_{\tilde{h}}=\frac{2}{b}(b-\tilde{h}-1)=1+\sqrt{1-\frac{4}{b}}>1,
$$

and we have a Neimark-Sacker bifurcation occurring at $h=\tilde{h}$. Finally, it is a computational matter to find that $\bar{x}_{\tilde{h}}^{3} f^{\prime \prime \prime}\left(\bar{x}_{\tilde{h}}\right)=-3 / 2$ at $b=4$ and increases in $b$. Thus, we invoke Theorem 4.2 to conclude that the invariant curve is supercritical. 

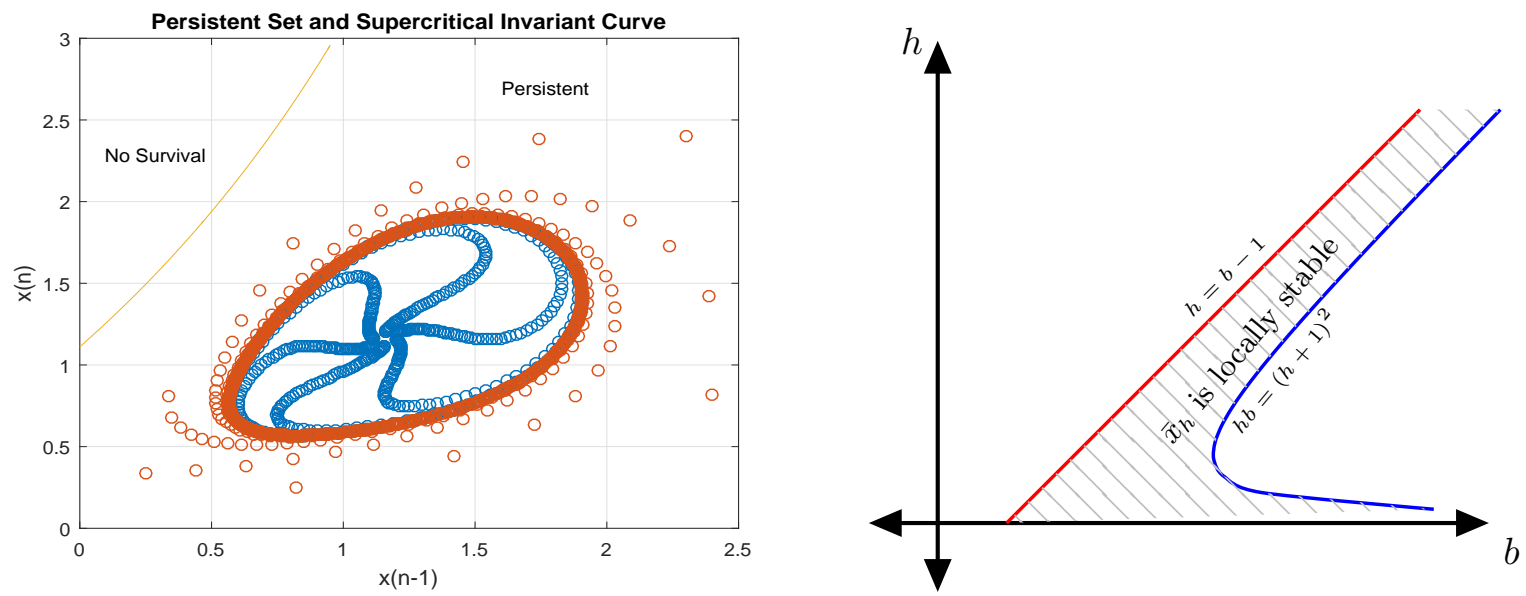

Figure 4: The graph on the left shows the strongly persistent set $\mathcal{D}_{h}^{*}$ at $b=4.1$ and $h=0.9$. The closed loop is the stable invariant curve obtained by the Namiark-Sacker bifurcation. Also, several orbits that converge to the stable curve from inside and outside are shown. The shaded region in the right graph $\left(h<b-1\right.$ and $\left.h^{2}+(2-b) h+1>0\right)$ shows the values of $(b, h)$ before obtaining the invariant curve in the right graph, and the blue curve on the right boundary represents the values of $(b, h)$ that give birth to the invariant curve on the right.

\section{Conclusion}

In this paper, we posed a general contest competition discrete-time model with and without delay in recruitment, which encompass several classical models such as Beverton-Holt [5] and Pielou [31,32], then we added a constant effort harvesting term, and explored the dynamics of the new model. At small harvesting level, the new model can encompass the dynamics of Shepherd [36] and Hassel [17] models. Adding harvesting term into the model had the effect of shrinking the persistent set, lead to period doubling then chaos when the delay is zero and to a supercritical invariant curve when the delay is one. At zero harvesting level, the persistent set is obviously the positive quadrant; however, as we increased the harvesting level, the persistent set required a closer look, which had been characterized. From an ecological point of view, increasing the harvesting level lead to changing the intraspecific competition from contest to scramble. This theoretical fact is in line with some experimental studies that show species exhibit different types of competition at different stages (see page 56 of [9] and the references therein).

Several aspects of the model are still under investigation. For example, in case of the delayed density dependence, it remains to show that local stability implies global stability with respect to the persistent set. This task needs the development of some theoretical as well as technical machinery, which is in fact the topic of some of our ongoing research. Also, other harvesting strategies can be considered and the impact on the dynamics of our general model can be investigated. 


\section{Acknowledgement}

The first author is partially supported by a seed grant offered by the American University of Sharjah.

\section{References}

[1] Z. AlSharawi and M. Rhouma, The Beverton-Holt model with periodic and conditional harvesting, Journal of Biological Dynamics 3(2009) 463-478.

[2] Z. AlSharawi and M. Rhouma, The Discrete Beverton-Holt Model with Periodic Harvesting in a Periodically Fluctuating Environment, Advances in Difference Equations, (2010), Article ID 215875, doi:10.1155/2010/215875.

[3] Z. AlSharawi and M. Rhouma, Coexistence and extinction in a competitive exclusion Leslie/Gower model with harvesting and stocking, Journal of Difference Equations and Applications 15 (2009) 1031-1053.

[4] T. S. Bellows JR, The descriptive properties of some models for density dependence, Journal of Animal Ecology 50 (1981) 139-156.

[5] Beverton, R. J. H. and Holt, S. J., On the Dynamics of Exploited Fish Populations, The Blackburn Press, New Jersey, 2004.

[6] L. Block and W. Coppel, Dynamics in One Dimension, Springer-Verlag, Berlin, 1992.

[7] F. Brauer, D. A. Sanchez, Constant rate population harvesting: Equilibrium and stability, Theoret. Population Biol. 8 (1975) 12-30.

[8] F. Brauer, D. A. Sanchez, Periodic environments and periodic harvesting, Natural Resources Modeling 16 (2003) 233-244.

[9] F. Brauer, C. Kribs, Dynamical Systems for Biological Modeling, CRC Press, UK, 2016.

[10] E. N. Brooks, J. Lebreton, Optimizing removals to control a metapopulation: application to the yellow legged herring gull (Larus cachinnans), Ecological Modelling, 136 (2001) 269-284.

[11] C. Castillo-Chavez, A. Yakubu, Intraspecific competition, dispersal and disease dynamics in discrete-time patchy environments, Mathematical approaches for emerging and reemerging infectious diseases: an introduction (Minneapolis, MN, 1999), 165-181, IMA Vol. Math. Appl. 125, Springer, New York, 2002.

[12] N. P. Chau, Destabilizing effect of periodic harvest on population dynamics, Ecological Modeling 127 (2000) 1-9.

[13] W. A. Coppel, The solution of equations by iteration, Proc. Cambridge Philos. Soc. 51 (1955) 41-43. 
[14] M. I. Costa, Harvesting induced fluctuations: Insights from a threshold management policy. Mathematical Biosciences 205 (2007) 77-82.

[15] S. Elaydi, R. Sacker, Basin of attraction of periodic orbits of maps on the real line, Journal of Difference Equations and Applications 10 (2004) 881-888.

[16] M. Fan, K. Wang, Optimal harvesting policy for single population with periodic coefficients. Mathematical Biosciences 152 (1998) 165-178.

[17] M. P. Hassel, Density dependence in single species populations, J. Animal Ecology 44 (1975) 283-295.

[18] J. Hale, H. Kocak, Dynamics and Bifurcation, Springer-Verlag, New York, 1991.

[19] Gert Van Der Heijden, Hopf bifurcation, Encyclopedia of Nonlinear Science, Alwyn Scott (ed.), Routledge, London and New York, 2004.

[20] S. M. Henson, J. M. Cushing, Hierarchical models of intraspecific competition: scramble versus contest, J. Math. Biol. (1996) 755-772.

[21] V. Hutson and W. Moran, Persistence of species obeying difference equations, J. Math. Biol. 15 (1982) 203-213

[22] M. R. S. Kulenović and G. Ladas, Dynamics of Second Order Rational Difference Equations with Open Problems and Conjectures, Chapman \& Hall/CRC, Boca Raton, 2002.

[23] Y. Kuznetsov, Elements of applied bifurcation theory, Springer-Verlag, 1998.

[24] E. Liz, How to control chaotic behavior and population size with proportional feedback, Physics Letters A 374 (2010) 725-728.

[25] E. Liz, F. M. Hilker, Harvesting and dynamics in some one-dimensional population models, Theory and Applications of Difference Equations and Discrete Dynamical Systems, Vol. 102 of the series Springer Proceedings in Mathematics \& Statistics, (2014) 61-73.

[26] E. Liz, A. Ruiz-Herrera, The hydra effect, bubbles, and chaos in a simple discrete population model with constant effort harvesting, J. Math. Biol. 65 (2012) 997-1016.

[27] A. Lomnicki, Scramble and contest competition, unequal resource allocation, and resource monopolization as determinants of population dynamics, Evolutionary Ecology Research 11 (2009) 371-380.

[28] A. Lomnicki, Population Ecology of Individuals, Princeton University Press, New Jersey, 1988.

[29] R. M. May, Simple mathematical models with very complicated dynamics, Nature 261 (1976) 459-467.

[30] A. J. Nicholson, An outline of the dynamics of animal populations, Australian Journal of Zoology 2 (1954) 9-65. 
[31] E. C. Pielou, An Introduction to Mathematical Ecology, Gordon and Breach, New York 1965.

[32] E. C. Pielou, Population and Community Ecology, Gordon and Breach, New York 1974.

[33] W. E. Ricker, Stock and Recruitment, J. Fish. Res. Bd. Can., 11 (1954) 559-623.

[34] S. Schriber, Chaos and population disappearances in simple ecological models, J. Math. Biol. 42 (2001) 239-260.

[35] H. Seno, A paradox in discrete single species population dynamics with harvesting/thinning, Math. Biosci. 214 (2008) 63-69.

[36] J. G. Shepherd, A versatile new stock-recruitment relationship for fisheries, and the construction of sustainable yield curves. J. Cons. Int. Explor. Mer, 40 (1982) 67-75.

[37] W. Van Den Berg, W. A. H. Rossing, J. Grasman, Contest and scramble competition and the carry-over effect in globodera spp. in potato-based crop rotations using an extended Ricker model, Journal of Nematology 38 (2006) 210-220.

[38] G.C. Varley, G.R. Gradwell, M.P. Hassel, Insect Population Ecology: an analytical approach, University of California Press, California, 1974.

[39] A. Yakubu, The effect of planting and harvesting on endangered species in discrete competitive systems, Mathematical Biosciences 126 (1994) 1-20.

[40] A. A. Yakubu, Two-patch dispersal-linked compensatory-overcompensatory spatially discrete population models, Journal of Biological Dynamics 1 (2007) 157-182.

[41] A. Yakubu, M. J. Fogarty, Periodic versus constant harvesting of discretely reproducing fish populations, Journal of Biological Dynamics 3 (2009) 342-356.

[42] E. F. Zipkin, C. E. Kraft, E. G. Cooch, P. J. Sullivan, When can efforts to control nuisance and invasive species backfire? Ecological Applications 19 (2009) 1585-1595. 